\title{
FRONTAL LOBE CONNECTIVITY AND COGNITIVE IMPAIRMENT IN FRONTAL LOBE EPILEPSY
}

Investigators at Maastricht University Medical Center, and Epilepsy Center Kempenhaeghe, The Netherlands, using functional magnetic resonance imaging (fMRI), studied the relationship between brain activation, functional connectivity, and cognitive functioning in 32 children aged 8-13 years with frontal lobe epilepsy (FLE) and 41 healthy age-matched controls. For the task-related fMRI, a Sternberg letter recognition task was used to induce cerebral activation, reflecting verbal working memory performance. Cognition was impaired in 16 children with FLE (50\%) and in 3 healthy controls (7\%). During working memory task performance, children with FLE showed a global decrease in functional brain connectivity compared to controls, whereas brain activation patterns remained intact. The widespread decrease in functional brain connectivity was similar in cognitively impaired and unimpaired patients. The decrease in frontal lobe connectivity in children with FLE complicated by cognitive impairment affected both connections within the frontal lobe and those from frontal to parietal and temporal lobes, cerebellum, and basal ganglia. The decrease in functional brain connectivity appeared to be related to the epilepsy itself, and was independent of cognitive performance.

The seizure types in this cohort of FLE patients were complex partial in $6(19 \%)$, atypical absence in $13(41 \%)$, and secondary generalized tonic-clonic in $5(15 \%)$. The seizure focus based on EEG and history was bifrontal in $17(53)$, left frontal in $8(25 \%)$ and right frontal in $7(22 \%)$. A history of febrile seizures was elicited in $9(28 \%)$ and status epilepticus in $2(6 \%)$. Seizures were refractory in $21(66 \%)$. (Braakman HMH, Vaessen MJ, Jansen JFA, et al. Frontal lobe connectivity and cognitive impairment in pediatric frontal lobe epilepsy. Epilepsia 2013 Mar;54(3):446-54). (Response: Dr Hilde MH Braakman, Department of Neurology, Maastricht University Medical Center, The Netherlands. E-mail: hilde.braakman@gmail.com).

COMMENT. Impairment of functional integrity of the frontal lobe network in children with FLE extends to connections to temporo-parietal lobes, cerebellum and basal ganglia. The relation between these altered functional networks and cognition in FLE is unexplained. The authors refer to a literature review of pediatric FLE and to functional connectivity studies in adult patients with mesial temporal lobe epilepsy showing altered connectivity in network structures distant from the seizure focus (Waites AB et al. Ann Neurol 2006 Feb;59(2):335-43). (Braakman HMH, et al. Cognitive and behavioral complications of frontal lobe epilepsy in children: a review of the literature. Epilepsia 2011 May;52(5):849-56).

\section{POPULATION-BASED STUDY OF EPILEPSY IN INFANTS}

Investigators at the Paediatric Neurology Department, Great Ormond Street Hospital for Children, London, and other centers in the UK and USA carried out a population-based study of children, 1-24 months of age, with new-onset epilepsy, ascertained over 13 months from 15 boroughs of North London. A total of 57 children were enrolled, an incidence of 70.1/100,000 children $<2$ years of age/year; 23 (41\%) were 
White, 21 (37.5\%) Asian, and 10 (18\%) Black. The risk was highest among Asian infants $(\mathrm{p}<0.001)$. An electroclinical syndrome was identified in $24(42 \%)$ cases of which 21 were epileptic encephalopathies (West [16], Ohtahara [2], and Dravet [3] syndromes). Overall, an underlying etiology for the epilepsy was identified in 29 children $(51 \%$ of the cohort). Developmental brain abnormalities (polymicrogyria, tuberous sclerosis) were most frequent, occurring in $11(21 \%)$, followed by acquired brain insults in $9(16 \%)$ infants. Acquired causes included meningitis in $5(9 \%)$, HIE in $4(7 \%)$, and metabolic disorder in 4 (7\%). Chromosomal abnormalities occurred in $4(7 \%)$. MR images of 51 cases showed abnormalities in $37(72 \%)$ and etiologically relevant abnormality in 26 (51\%). (Eltze CM, Chong WK, Cox T, et al. A population-based study of newly diagnosed epilepsy in infants. Epilepsia 2013 Mar;54(3):437-445). (Response: Dr Christin M Eltze, Neurosciences Unit, UCL-Institute of Child Health, 4/5 Long Yard, London WC1N 3LU, UK. E-mail: c.eltze@ucl.ac.uk).

COMMENT. Infantile onset epilepsy frequently presents with intractable seizures and is commonly associated with diffuse encephalopathy, metabolic or structural brain abnormalities. Identification of specific electroclinical syndromes at seizure onset requires specialist intervention with video-EEG recordings, MRI, metabolic, and genetic studies. The EEG associated with an epileptic encephalopathy is diffusely abnormal and varies with cerebral maturation. (Nordli DR Jr. Epileptic encephalopathies in infants and children. J Clin Neurophysiol 2012 Oct;29(5):420-4). Details of EEG findings are important in the workup and in the classification of infantile seizures. Early referral of infantile seizure patients to a Pediatric Epilepsy Center is usually indicated for accurate seizure classification and optimal management. (Alam S, Lux AL. Epilepsies in infancy. Arch Dis Child 2012 Nov;97(11):985-92).

\section{GENETICS OF BENIGN FAMILIAL INFANTILE EPILEPSIES}

Investigators at Instituto G Gaslini, Genova, Italy and multiple other centers in Italy studied the genetics of benign familial epilepsies of the first year of life and assessed the extent of the genetic overlap between neonatal and infantile seizure syndromes. Families with at least two first-degree relatives affected by focal seizures with onset in the first year of life and normal development before seizure onset were included. A total of 46 families including 165 affected members were collected and were classified as benign familial neonatal seizures (BFNS) in 8 families, benign familial neonatal-infantile seizures (BFNIS) in 9 (1-4 months of age at onset), and benign familial infantile seizures (BFIS) in 29 (onset after 4 months of age in all family members). Genetic analysis identified 41 mutations, 14 affecting KCNQ2, 1 affecting KCNQ3, 5 affecting SCN2A, and 21 affecting PRRT2. The detection rate of mutations in this cohort was $89 \%$. Mutations specifically involve KCNQ2 in BFNS, KCNQ2 (6 families) and SCN2A (two families) in BFNIS. BFIS families are most genetically heterogeneous, with all 4 genes involved, $70 \%$ carrying a PRRT2 mutation. PRRT2 mutations are clustered in families with BFIS and also, with paroxysmal kinesigenic dyskinesia.

KCNQ2 mutation is frequently represented in the entire spectrum of disorders, progressively decreasing with age, and may be predictive of afebrile seizures during follow-up, beyond the typical neonatal seizures. Age of onset of seizures is significantly 